\title{
Devra-t-on changer notre parc de coloscopes pour le dépistage en 2015 ?
}

\author{
Should our Type of Colonoscope be Changed for Screening in 2015 ?
}

\section{Heresbach}

(C) Lavoisier SAS 2014

La coloscopie est associée à un taux de faux-négatifs variant de 3,8 et $20 \%$ pour les adénomes avancés, de 6-9 $\mathrm{mm}$ ou inférieurs à $5 \mathrm{~mm}$. Ce taux de faux-négatifs est observé lors des essais dédiés à cette question soulignant le défaut technique des matériels disponibles. Plusieurs techniques ont été proposées comme la coloscopie avec un cap transparent, le recours à un troisième œil et plus simplement une durée optimale de retrait du coloscope (deux minutes par segment, soit $\geq 8$ minutes) ou une préparation décrite comme excellente (score de Boston $>7$ ou de Harefield $\mathrm{A}$ ou $\mathrm{B}$ ). Après une étude de faisabilité, l'article de Gralnek et al. [1] décrit la sensibilité de la coloscopie avec vision quasi circulaire $\left(330^{\circ}\right)$ grâce à un coloscope et processeur permettant la visualisation sur trois écrans soit la vision axiale classique et en plus les visions latérales droite et gauche. Ce système de Full Spectrum Endoscopy ou FUSE est possible grâce au recours à un éclairage non pas réalisé par des fibres mais obtenu par les diodes ou LED axiales et latérales. Ainsi, ce coloscope a un diamètre de 12,8 $\mathrm{mm}$ avec un canal opérateur de $3,8 \mathrm{~mm}$, réutilisable comme les coloscopes classiques et son angle de vision est de $170^{\circ}$. Dans un essai contrôlé randomisé multicentrique, un groupe de 88 patients étaient explorés en premier par un coloscope classique, puis avec le système FUSE alors que le second groupe de 97 patients avaient les deux examens dans l'ordre inverse. Les deux groupes étaient comparables bien que le temps d'endoscopie et de retrait était plus allongé avec le système FUSE (12,2 versus $14,5$ minutes, $p<0,001)$. Dans ces deux groupes, le taux d'omission des adénomes était respectivement de 41 versus $7 \%(p<0,0001)$ dont trois adénomes avancés dans le premier groupe (omis par coloscopie classique). En analyse par patient, parmi les 88 patients, cinq $(6 \%)$ avaient un adénome visualisé par le système FUSE et omis par la coloscopie classique, alors qu'aucun «nouveau patient» n'était porteur d'un adénome omis par le système FUSE. Cette étude ne précise pas la taille des 20 et 5 adénomes omis respectivement dans ces deux groupes mais la reproductibilité de ces résultats aurait une conséquence non négligeable sur les équipements des plateaux d'endoscopie. En effet, le processeur est spécifique ou système FUSE qui n'est pas un simple traitement électronique de l'image ou du spectre lumineux obligeant à l'acquisition d'un nouvel équipement.

Conflit d'intérêt : Le Pr Denis Heresbach a réalisé des missions ponctuelles d'expert auprès des laboratoires Norgine, Aptalis en 2010 et de la société MedPass, Norgine et Aptalis en 2011. Il a participé à un symposium organisé par MaunaKea Technology en 2010, Ella SA en 2011 et au Club francophone d'échoendoscopie (CFE) de 2012 avec le soutien de Wilson-Cook France.

\section{Référence}

1. Gralnek IM, Siersema PD, Halpern Z, Segol O, Melhem A, Suissa A, et al. Standard forward-viewing colonoscopy versus full-spectrum endoscopy: an international, multicentre, randomised, tandem colonoscopy trial. Lancet Oncol 2014; 15:353-60.

\footnotetext{
D. Heresbach $(\bowtie)$

Unité d'endoscopie et de consultation digestive, centre hospitalier de Redon, 8, rue Étienne-Gascon, F-35603 Redon, France

e-mail : Denis.heresebach@ch-redon.fr
} 